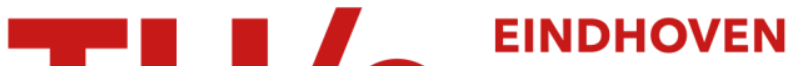 UNIVERSITY OF TECHNOLOGY
}

\section{Particle-based modeling of heterogeneous chemical kinetics including mass transfer}

\section{Citation for published version (APA):}

Sengar, A., Kuipers, J. A. M., van Santen, R. A., \& Padding, J. T. (2017). Particle-based modeling of heterogeneous chemical kinetics including mass transfer. Physical Review E, 96(2), [022115]. https://doi.org/10.1103/PhysRevE.96.022115

DOI:

10.1103/PhysRevE.96.022115

Document status and date:

Published: 08/08/2017

\section{Document Version:}

Publisher's PDF, also known as Version of Record (includes final page, issue and volume numbers)

\section{Please check the document version of this publication:}

- A submitted manuscript is the version of the article upon submission and before peer-review. There can be important differences between the submitted version and the official published version of record. People interested in the research are advised to contact the author for the final version of the publication, or visit the $\mathrm{DOI}$ to the publisher's website.

- The final author version and the galley proof are versions of the publication after peer review.

- The final published version features the final layout of the paper including the volume, issue and page numbers.

Link to publication

\section{General rights}

Copyright and moral rights for the publications made accessible in the public portal are retained by the authors and/or other copyright owners and it is a condition of accessing publications that users recognise and abide by the legal requirements associated with these rights.

- Users may download and print one copy of any publication from the public portal for the purpose of private study or research.

- You may not further distribute the material or use it for any profit-making activity or commercial gain

- You may freely distribute the URL identifying the publication in the public portal.

If the publication is distributed under the terms of Article 25fa of the Dutch Copyright Act, indicated by the "Taverne" license above, please follow below link for the End User Agreement:

www.tue.nl/taverne

Take down policy

If you believe that this document breaches copyright please contact us at:

openaccess@tue.nl

providing details and we will investigate your claim. 


\title{
Particle-based modeling of heterogeneous chemical kinetics including mass transfer
}

\author{
A. Sengar* and J. A. M. Kuipers \\ Department of Chemical Engineering and Chemistry, Eindhoven University of Technology, \\ P.O. Box 513, 5600 MB Eindhoven, The Netherlands
}

Rutger A. van Santen

Institute for Complex Molecular Systems, Eindhoven University of Technology, P.O. Box 513, 5600 MB, Eindhoven, The Netherlands

J. T. Padding ${ }^{\dagger}$

Process and Energy Department, Delft University of Technology, Leeghwaterstraat 39, 2628 CB, Delft, The Netherlands

(Received 24 May 2017; published 8 August 2017)

\begin{abstract}
Connecting the macroscopic world of continuous fields to the microscopic world of discrete molecular events is important for understanding several phenomena occurring at physical boundaries of systems. An important example is heterogeneous catalysis, where reactions take place at active surfaces, but the effective reaction rates are determined by transport limitations in the bulk fluid and reaction limitations on the catalyst surface. In this work we study the macro-micro connection in a model heterogeneous catalytic reactor by means of stochastic rotation dynamics. The model is able to resolve the convective and diffusive interplay between participating species, while including adsorption, desorption, and reaction processes on the catalytic surface. Here we apply the simulation methodology to a simple straight microchannel with a catalytic strip. Dimensionless Damkohler numbers are used to comment on the spatial concentration profiles of reactants and products near the catalyst strip and in the bulk. We end the discussion with an outlook on more complicated geometries and increasingly complex reactions.
\end{abstract}

DOI: 10.1103/PhysRevE.96.022115

\section{INTRODUCTION}

Many of the chemicals and fuels encountered in our daily life are products of chemical conversion in reactors, where catalyst materials are used to accelerate the conversion rate without being consumed themselves. Heterogeneous catalysis, i.e., reactions taking place on the surface of a solid catalytic material (deposited on or inside a structured or porous support) is the most common type of catalytic conversion. The design and optimization of heterogeneous catalytic reactors is an intrinsically multiscale and multiphysics problem: the microscale (intrinsic) kinetics are determined by quantum chemical effects, while the macroscale (effective) kinetics are often limited by flow and diffusion of reactant and product species around and through the support structures. A number of computational methods have been developed to capture these effects. Density functional theory modeling $[1,2]$ is one such approach to study molecular electronics, whereas microkinetic modeling [3] focuses on the surface chemistry of reactions with the aim of generating rate constants for different reaction pathways that lead to product generation. At the macroscale, the discrete element method [4] and direct numerical simulations [5] model large multicomponent systems by looking at the particle interactions and resolving the hydrodynamic forces surrounding these particles. The connection between these two scales is critical as information needs to be passed from the microworld in some usable form to the macroworld, and this is where the need for the mesoscale world emerges with a smooth transition from a discrete environment to the continuum.

\footnotetext{
*a.sengar@tue.nl

$\dagger$ j.t.padding@ tudelft.nl
}

Various mesoscale techniques could be used such as the Lattice-Boltzmann method, direct simulation Monte Carlo, and dissipative particle dynamics. One approach that may be particularly suitable for the study of reactive surfaces coupled to diffusive flows is stochastic rotation dynamics (SRD), originally developed in 1999 by Malevanets and Kapral [6]. SRD relies on the discrete-time dynamics of coarse-grained particles in a continuous space, executing computationally efficient local collisions of particles with their neighbours while conserving momentum and energy [7]. Given that SRD is a stochastic approach, thermal fluctuations are inherently present in the calculation of any dependent variable. The automatic inclusion of hydrodynamic interactions, both shortrange and longe-range, gives the model a very appealing tone. The transport process variables have been extensively studied, and analytical expressions to describe them have previously been derived in Refs. [8-14]. SRD has been implemented to study various systems such as polymer flow $[15,16]$, phase separation in binary and tertiary mixtures [17], colloidal suspensions [18], vesicles in shear flow [19], enzyme catalysis [20], and anisotropic interaction in liquid separation [21].

The SRD model has been extended to include homogeneous reactions. Rohlf et al. [22] used the approach by including birth-death stochastic processes to study limit cycle formation in the Selkov model. The approach developed is called the reactive multiparticle collision model. The SRD model has also been extended to simple heterogeneous reactions occurring on the surface of a spherical catalyst particle [23-26]. In these works, it is assumed that the reaction happens instantaneously with a certain probability once the reactant species collides with the catalytic sphere. The method has first been applied to static catalytic particles $[23,24]$ and later extended to self-propelled nanomotors [25,26]. This method applies to 
instantaneous reactions that are only transport limited and not reaction limited.

Most real heterogeneous chemical systems have certain rate-determining steps associated with the individual processes, such as adsorption, desorption, and individual elementary reaction steps that require overcoming large activation barriers. The aim of this paper is to show that SRD can also be used to study such multicomponent systems involving various time scales associated with both transport and reaction limitations. Other particle-based techniques have been previously used to study reaction-diffusion systems [27], but the introduction of a surface reaction model that incorporates different reaction rates for each participating process in this work extends the model beyond previous studies. Squires et al. [28] have developed a first-order adsorption desorption model under quasisteady-state equilibrium conditions. The present model will expand further on the model of Squires et al. by giving the adsorbed species different pathways of desorption and reaction.

The paper is arranged as follows. In Sec. II we focus on the development of the model, stating the presence of catalyst inside the system, interaction of the reactants with this catalyst leading to product formation, and diffusion of these products out of the system. This study also involves determining rate parameters used to construct the system. In Sec. III we describe the surface reaction models and provide a dimensionless analysis to compare the diffusive and reactive fluxes at a steady state. Damkohler numbers [29] are defined to draw out this comparison. In Sec. IV we validate the model for (a) diffusion by comparing the simulation results with the analytical solution for a convection-diffusion problem and (b) reactions by comparing equilibrium surface coverage over the catalyst with the solution for the surface reaction model. A new adsorption and desorption technique for a particle-based model is mentioned that can be expanded to include reactions. In Sec. $\mathrm{V}$ we study the temporal and spatial concentration profiles of product species generated for different Damkohler numbers. Finally, we draw conclusions and present an outlook for further studies with the model in Sec. VI.

\section{METHODOLOGY}

The solvent present in SRD is represented by a coarsegrained ensemble of fluid point particles. There has been sufficient discussion with regard to approaches to model a pure solvent model using SRD [13,18]. Inclusion of different species with different diffusivities makes the dynamics more interesting with regard to real world applicability.

\section{A. Fluid model}

In this paper we will focus on a simple narrow slitlike microfluidic reactor, described in more detail in the next subsection. The participating species in the model reactor are modeled as follows. Inside the system, $N$ particles are placed, such that the total mass in the reactor is $\sum_{i=1}^{k} \chi_{i} m_{i} N$ where $\chi_{i}$ and $m_{i}$ are the mole fraction and mass of component $i$, respectively, and $k$ is the number of components inside the system. In the scope of this study, $k$ will be either 1 or 2 .
The two steps for the SRD algorithm are the streaming and the collision step. During the streaming step, for every discrete time interval $\Delta t_{c}$, the coordinates are updated using a first-order Euler scheme

$$
\mathbf{r}_{i}^{t+1}=\mathbf{r}_{i}^{t}+\mathbf{v}_{i}^{t} \Delta t_{c}
$$

where $\mathbf{r}_{\mathbf{i}}{ }^{t}$ and $\mathbf{v}_{\mathbf{i}}{ }^{t}$ are the position and velocity vector of particle $i$ at time $t$. The particles do not interact with each other during the streaming step. For the collision step, the three-dimensional space is coarse grained into a grid of cubic lattice cells of size $a_{0}$. Interparticle collisions are then carried out within each cell space where the particle velocity is updated using the expression

$$
\mathbf{v}_{i}^{t+1}=\overline{\mathbf{v}}+\boldsymbol{\Omega}\left(\mathbf{v}_{i}^{t}-\overline{\mathbf{v}}\right) .
$$

Here $\overline{\mathbf{v}}$ is the center-of-mass velocity of all particles in the same cell as particle $i$ and therefore takes into account the presence of different kinds of particles, and $\boldsymbol{\Omega}$ is a stochastic rotation matrix that rotates the velocity vector of each particle in the center of mass reference frame around a randomly oriented axes by a fixed angle $\alpha$. It has been shown previously [6] that there is an $H$ theorem to the algorithm stating that the velocities equilibrate to a Maxwell distribution, and that the macroscopic behavior is in accordance with the hydrodynamic (NavierStokes) equations conforming to the ideal-gas equation of state.

A random grid cell shift procedure is employed just before the collision procedure to ensure Galiliean invariance [8]. If the mean-free path of particles is more than half the grid cell size, this shift procedure is not required as the particles undergo momentum transfer both within and outside the grid cells in subsequent time steps.

Due to the grid-shift procedure, certain cells at the boundary will be underfilled and certain cells will be overfilled. Lamura et al. [30] first mentioned the introduction of pseudoparticles to account for the possible artificial lower viscosity of boundary cells. This was further extended by Whitmer and Luijten [31] for overfilled boundary cells. Appendix A provides further details about the treatment of boundary cells in a multicomponent system.

In some cases a body force is used to drive a flow in the fluid. In such a case, the velocity update by the collision step, Eq. (2) is immediately followed by a change in velocity $\mathbf{v}_{i}^{t+1}=\mathbf{v}_{i}^{t+1}+$ $\mathbf{g} \Delta t_{c}$, where $\mathbf{g}$ is the acceleration associated with this body force. Note that care must be taken to not apply a large body force to stay away from Mach number limitations caused by the high compressibility of the SRD fluid [18]. Also, the external body force will supply energy to the system, which will result in an overall increment in the energy of the system, leading to an increase in the average temperature. A Galilean invariant thermostat proposed by Padding and Louis [18] has therefore been implemented. The thermostat performs a global rescaling of the velocity fluctuations in the center of mass frame to calibrate the temperature for the next time step. Furthermore, no-slip boundaries are included such that any particle that reflects off a boundary surface experiences a velocity reversal (also called bounce-back). This ensures that particles have a net zero velocity adjacent to the boundaries for long-time steady-state averages. 
TABLE I. Simulation parameters used and their units.

\begin{tabular}{|c|c|}
\hline Unit & Expression \\
\hline Length & $a_{0}$ \\
\hline Energy & $k_{B} T$ \\
\hline Mass of solvent & $m_{f}$ \\
\hline Time & $t_{0}=a_{0} \sqrt{\frac{k_{B} T}{m_{f}}}$ \\
\hline Diffusion coefficient & $D_{0}=\frac{a_{0}^{2}}{t_{0}}=a_{0} \sqrt{\frac{k_{B} T}{m_{f}}}$ \\
\hline $\begin{array}{l}\gamma: \text { average number of } \\
\Delta t_{c}: \text { SRD streaming ti } \\
\alpha: \text { SRD rotation angle } \\
W, H, L: \text { Length of bo }\end{array}$ & s \\
\hline
\end{tabular}

The dimensionless mean-free path $\lambda$ is important when considering characteristic length scales. In our simulations units, shown in Table I, the dimensionless mean-free path [18] equals $\lambda=\Delta t_{c} / t_{0}$.

The diffusion coefficient for a single particle in a pure solvent has been calculated by Kapral [32]:

$$
\frac{D}{D_{0}}=\frac{k_{B} T \Delta t_{c}}{2 m_{f}}\left[\frac{3 \gamma}{\left(\gamma-1+e^{-\gamma}\right)(1-\cos \alpha)}-1\right] .
$$

This equation is accurate for larger mean-free paths $\lambda>0.6$. However, deviations occur for smaller mean-free paths when long-time kinetic correlations develop. In such a case, the probability of two or more particles occupying the same grid cell at subsequent time steps increases rapidly leading to an underestimation of the diffusion coefficient given by Eq. (3). The corrections have been studied by Ripoll et al. [16].

\section{B. Reactor model}

Introduction of a binary mixture in this reactor will pave the way for the study of multicomponent flows in addition to chemical reactions where a product formation is facilitated by the presence of a catalytic strip in the system that leads to concentration gradients of reactant and product components. Since the aim of the paper lies in validating the use of SRD model to carry out reactions, a study of a simple reaction $A \rightarrow B$ will be undertaken when $m_{A}=m_{B}$. The diffusion coefficient of both the components is therefore the same, as can be seen from Eq. (3). The interactions between the different species is assumed to be ideal, as is the case for an ideal gas system. Extensions to nonideal interactions are studied by Tuzel et al. [17] for phase separation behavior in a mixture but are not implemented in this work. Including complex reactions where reactants and products have different masses and different mobilities will be the topic of a future publication.

Figure 1 shows a schematic of the reactor model. The simulation domain is rectangular, with dimensions $W, H$, and $L$. Periodic boundaries are applied along the $x$ axis and $z$ axis. If a product particle, $\mathrm{B}$, crosses over the $z$-axis boundary, it converts into a reactant molecule, $\mathrm{A}$, while conserving mass. For the $x$-axis boundary crossover, only the particle position is updated. Walls are placed along the $y$ axis at

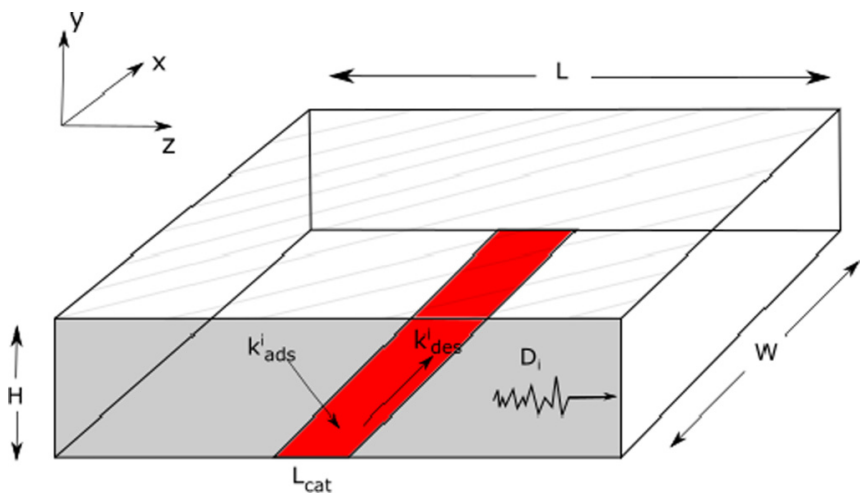

FIG. 1. The catalyst is present at the center of the system with dimensions $L_{\text {cat }} \times W$, The kinetic rate constants for the first-order Langmuir reaction for particle of type $i$ are $k_{\text {ads }}^{i}$ and $k_{\text {des }}^{i}$ and the diffusivity of particle of type $i$ is $D_{i}$.

$y=0$ and $y=H$. The system is initialized by filling the space with the reacting solvent, A, with a number density $\gamma$. The particle coordinates are chosen at random, with velocity fluctuations from a Maxwell-Boltzmann distribution with a standard deviation of $\sqrt{k_{b} T / m_{A}}$. For longer time averages, the net velocity will always be zero, unless an external body force is applied. At $y=0$, a region $L_{\text {cat }}$ in width and $W$ in length at the center of the $z$ axis is defined as the catalytic strip. This strip contains $N_{\text {cat }}$ open positions to collect particles. Any particle that bounces on the strip has a probability $p_{\text {on }}$ of attaching to the strip, i.e., the adsorption probability is controlled by $p_{\text {on }}$. The probability parameter is a measure of the enthalpy of activation for adsorption and arises from the Eyring-Polanyi equation in Transition State theory [33]. Having encountered the wall, if the reactant particle does not meet the strip or does not stick to it within that time step, it is reflected back with a bounce-back rule to maintain a no-slip condition at the wall [7].

For any adsorption of A occurring on the surface, the position of the particle on the strip is recorded. After adsorption, the particle has three choices. There is a probability of desorption, $p_{\text {des }}$, that the particle leaves the surface, a probability $p_{r+}$ that it reacts to form a product particle $\mathrm{B}$ (which might further desorb from the surface), and a probability $1-p_{\text {des }}-p_{r+}$ that it stays adsorbed. Note that the probabilities $p_{\text {des }}$ and $p_{r+}$ apply to a single time step $\Delta t_{c}$. We will relate them to a rate constant in the next subsection. When the particle desorbs, it desorbs from the recorded location, which is an accurate approximation for diffusion-limited surface reactions. Particles desorbing from the catalytic strip obtain a tangential and a normal velocity component, the distribution of which is equivalent to the velocity distributions of incoming particles that adsorbed on the strip. This ensures reversibility in the system with respect to the reactant and product species. Further details concerning the treatment of adsorption and desorption at the catalytic strip for different mass particles are presented in Appendix B.

Once product particles (B) are desorbed back inside the bulk of the system, they diffuse towards the ends of the domain along the $z$ direction, from where they are taken out of the system. In other words, at $z=0$ and $z=L$, there is a reservoir consisting of only particles of type $\mathrm{A}$, and the concentration of B falls down to zero. 


\section{Linking probabilities to intrinsic rates}

Implementing adsorption rates in a probabilistic manner for either microscale or macroscale models has been shown previously in Ref. [34]. Desorption is usually a chemically activated step while adsorption takes place with comparative ease. The rate constant for such processes can be determined from the Eyring-Polanyi equation. The energy barrier determines the thermodynamic ease and the pre-exponential factor determines the kinetic ease of carrying out either of the processes. The adsorption-desorption process will equilibrate with a constant $K_{\text {eq }}=k_{\text {ads }} / k_{\text {des }}$ that determines the occupancy of the available catalyst surface sites. Here $k_{\text {ads }}$ is the adsorption rate constant and $k_{\text {des }}$ is the desorption rate constant for a first-order adsorption-desorption scheme to and from the catalytic strip, respectively.

We now calculate these intrinsic rates in terms of the SRD simulation parameters we have discussed earlier in this section. Pooley and Yeomans [10] have previously used a kinetic theory approach in SRD to calculate viscosity and thermal conductivity expressions. Here we extend the kinetic model of an ideal gas to derive an expression for the collisional rate at walls. The collisional frequency, $Z$, i.e., the number of collisions occurring per unit area of the wall per unit time step, has been shown in Ref. [35] to be

$$
Z=\frac{1}{2} \frac{N}{V}\left\langle\left|v_{y}\right|\right\rangle,
$$

where $N$ is the total number of particles inside the system, $V$ is the volume, and $\left\langle\left|v_{y}\right|\right\rangle$ is the first moment of the velocity component of particles perpendicular to the wall. The above expression is valid for a noninteractive surface where no adsorption occurs on the wall, thus the number density $\mathrm{N} / \mathrm{V}$ is constant throughout the system. When adsorption occurs, $N / V$ should be replaced by $C_{s}=\gamma_{s}$, the local concentration of particles near the surface. $C_{s}$ will be a function of time when adsorption over the catalyst starts and will be so until the reaction attains a steady state. The use of the expression $C_{s}$ rather than $\gamma_{s}$ in the follow-up sections is motivated by the terminology followed when dealing with reaction kinetics. Since the velocity distribution of the particles evolves to a Maxwell-Boltzmann distribution [6], the average velocity expectation is

$$
\left\langle v_{y}\right\rangle=\sqrt{\frac{2 k_{b} T}{\pi m}} .
$$

Here $m$ is the mass of the component species that is under consideration.

Combining Eq. (4) and Eq. (5) leads to

$$
Z=C_{s} \sqrt{\frac{k_{b} T}{2 \pi m}} .
$$

The dimensions of $Z$ are $a_{0}^{-2} t_{0}^{-1}$, or in SI, $\mathrm{m}^{-2} \mathrm{~s}^{-1}$. If the total area of the catalyst surface is $A_{\text {cat }}$, the number of catalytic sites is $N_{\text {cat }}$, and the probability that a striking particle sticks is $p_{\text {on }}$, the attacking rate $k_{\text {on }}$ can be written as

$$
k_{\mathrm{on}}=\frac{p_{\mathrm{on}} A_{y} Z}{N_{\mathrm{cat}}}=C_{s} \sqrt{\frac{k_{b} T}{2 \pi m}} \frac{p_{\mathrm{on}} A_{y}}{N_{\mathrm{cat}}}
$$

The dimensions of $k_{\mathrm{on}}$ are $t_{0}^{-1}$, or in SI, $\mathrm{s}^{-1}$. The adsorption rate constant, with dimensions $\mathrm{m}^{3} \mathrm{~s}^{-1}$, can be found by dividing out the local concentration of particles near the surface:

$$
k_{\mathrm{ads}}=\sqrt{\frac{k_{b} T}{2 \pi m}} \frac{p_{\mathrm{on}} A_{\mathrm{cat}}}{N_{\mathrm{cat}}} .
$$

To derive an expression for $k_{\mathrm{des}}$, the desorption rate, consider an adsorbed particle on the catalytic surface. The probability that such a particle does not desorb after $n \Delta t_{c}$ time steps is $\left(1-p_{\text {des }}\right)^{n}$. So with a probability $1-\left(1-p_{\text {des }}\right)^{n}$, the particle can desorb at any time between 0 and $n \Delta t_{c}$. Since the particle desorption is usually a first-order process [36], the first-order desorption of a particle from the surface is exponential with a decay rate $k_{\mathrm{des}}$. Thus, for a continuum process, the probability that a particle will desorb anytime between 0 and $n \Delta t_{c}$ is $1-e^{-k_{\operatorname{des}} n \Delta t_{c}}$. Equating the discrete and continuum probabilities leads to

$$
1-\left(1-p_{\mathrm{des}}\right)^{n}=1-e^{-k_{\operatorname{des}} n \Delta t_{c}},
$$

from which the desorption rate $k_{\text {des }}$ is given as

$$
k_{\mathrm{des}}=-\frac{\ln \left(1-p_{\mathrm{des}}\right)}{\Delta t_{c}} .
$$

A similar analysis can be done for the reaction rate to obtain

$$
k_{r+}=-\frac{\ln \left(1-p_{r+}\right)}{\Delta t_{c}} .
$$

The above analysis has been done in general for either of the reactor or product particles. Any probability or the consecutive rate constant for a specific component $i$ from now will be represented by using a superscript $i$.

\section{SURFACE REACTION MODELS}

Having described the multicomponent fluid and reactor models, we now introduce a simple adsorption-desorptionreaction scheme which extends the RMPC model for heterogeneous catalysis beyond the assumption of an instantaneous reaction. Our model can therefore cover the the full spectrum from simple first-order to highly nonlinear reactions.

\section{A. Langmuir model}

For a first-order Langmuir model, detailed equilibrium expressions of occupancy of a catalytic surface have been previously derived in Refs. [37,38]. With the knowledge of the rate parameters described in Sec. II B 1, we can now solve the Langmuir model with the following rate equation for fractional occupancy of A on the surface given by

$$
d \theta_{A} / d t=k_{\mathrm{ads}}^{A} C_{s}^{A} \theta-k_{\mathrm{des}}^{A} \theta_{A},
$$

where $\theta_{A}(t)$ is the fraction of adsorbed particles $A$ at the surface and $\theta=1-\theta_{A}$ is the fraction of unbounded sites available for adsorption. The solution to Eq. (12) is

$$
\theta_{A}(t)=\theta_{A, \mathrm{eq}}\left(1-e^{-\left(k_{\mathrm{ads}}^{A} C_{s}^{A}+k_{\mathrm{des}}^{A}\right) t}\right),
$$

where $\theta_{A, \text { eq }}$ is the occupancy at equilibrium

$$
\theta_{A, \mathrm{eq}}=\frac{K_{\mathrm{eq}}^{A} C_{s}^{A}}{1+K_{\mathrm{eq}}^{A} C_{s}^{A}}
$$


TABLE II. Independent, dependent, and derived parameters in the model.

\begin{tabular}{lc}
\hline \hline Input parameter & Expression \\
\hline Sticking probability & $p_{\mathrm{on}}^{i}$ \\
Desorption probability & $p_{\mathrm{des}}^{i}$ \\
Reaction probability & $p_{r+}$ \\
Bulk concentration & $C_{0}^{i}$ \\
\hline Output parameter & Expression \\
\hline Local concentration above the catalytic strip & $C_{s}^{i}$ \\
Fractional surface coverage of component $i$ & $\theta_{i}$ \\
on catalyst & $\theta$ \\
Fractional vacancy on catalyst & $\operatorname{Expression}$ \\
\hline Derived rate constants & $k_{\mathrm{ads}}^{i}=\sqrt{\frac{k_{b} T}{2 \pi m_{i}}} \frac{p_{\mathrm{on}}^{i} A_{\mathrm{cat}}}{N_{\mathrm{cat}}}$ \\
\hline Adsorption rate & $k_{\mathrm{des}}^{i}=\ln \left(1-p_{\mathrm{des}}^{i}\right) / \Delta t_{C}$ \\
Desorption rate & $k_{r+}=\ln \left(1-p_{r+}\right) / \Delta t_{C}$ \\
Reaction rate & $K_{\mathrm{eq}}^{i}=k_{\mathrm{ads}}^{i} / k_{\mathrm{des}}^{i}$ \\
\hline Equilibrium constant
\end{tabular}

and $K_{\mathrm{eq}}^{A}=k_{\mathrm{ads}}^{A} / k_{\mathrm{des}}^{A}$. Table II summarizes the independent and dependent parameters used in the model. When diffusion in the fluid facilitates faster transport of particles to the surface than adsorption and desorption can occur, $C_{s}^{A} \sim C_{0}^{A}$ (concentration near the surface is equal to concentration in the bulk).

The total rate of the combined phenomena is equal to $k_{\text {tot }}^{A}=$ $k_{\mathrm{ads}}^{A} C_{s}^{A}+k_{\mathrm{des}}^{A}$ and in terms of time scales, $1 / \tau_{\mathrm{tot}}^{A}=1 / \tau_{\mathrm{ads}}^{A}+$ $1 / \tau_{\text {des }}^{A}$, where $\tau_{\text {ads }}^{A}, \tau_{\text {des }}^{A}$ are the time scales associated with adsorption and desorption for the A particles. The same model has been used by Squires et al. [28] to study surface reactions, in a quasisteady state, within a reactor with flow. Here we will expand on the model by allowing for the study of the temporal and spatial evolution as it approaches equilibrium.

\section{B. Langmuir Hinshelwood model}

The Langmuir model can be extended to include a chemical reaction once a particle is adsorbed on to the catalytic surface. Consider the following Langmuir Hinshelwood reaction model [39]:

$$
A \underset{k_{\mathrm{des}}^{A}}{\stackrel{k_{\mathrm{dus}}^{A}}{\rightleftharpoons}} A * \stackrel{k_{r+}}{\longrightarrow} B * \underset{k_{\mathrm{on} B}}{\stackrel{k_{\mathrm{des}}^{B}}{\rightleftharpoons}} B .
$$

The rate equations for this model are

$$
\begin{aligned}
& \frac{d \theta_{A}}{d t}=k_{\mathrm{ads}}^{A} C_{s}^{A} \theta-k_{\mathrm{des}}^{A} \theta_{A}-k_{r+} \theta_{A}, \\
& \frac{d \theta_{B}}{d t}=k_{r+} \theta_{A}-k_{\mathrm{des}}^{B} \theta_{B}+k_{\mathrm{ads}}^{B} C_{s}^{B} \theta,
\end{aligned}
$$

where $\theta_{A}$ and $\theta_{B}$ are the surface coverages of adsorbed particles $\mathrm{A}$, referred to as $\mathrm{A}^{*}$, and adsorbed particles $\mathrm{B}$, referred to as $\mathrm{B}^{*}$, on the catalytic strip, respectively. $k_{r+}$ is the rate constant for the forward conversion reaction. $k_{\mathrm{ads}}^{i}$ and $k_{\mathrm{des}}^{i}$ are the adsorption and desorption rate constants.
The steady-state coverage for $\theta_{A}$ and $\theta_{B}$ can be calculated by solving Eq. (15) for long-time estimates:

$$
\begin{aligned}
& \theta_{A, \mathrm{eq}}=\frac{K_{\mathrm{eq}}^{A} C_{s}^{A}}{1+K_{\mathrm{eq}}^{A} C_{s}^{A}+K_{\mathrm{eq}}^{B} C_{s}^{B}+k_{r+}\left(\frac{K_{\mathrm{eq}}^{A} C_{s}^{A}}{k_{\mathrm{des}}^{B}}+\frac{K_{\mathrm{eq}}^{B} C_{s}^{B}}{k_{\mathrm{des}}^{A}}+\frac{1}{k_{\mathrm{des}}^{A}}\right)} \\
& \theta_{B, \mathrm{eq}}=\frac{K_{\mathrm{eq}}^{B} C_{s}^{B}+k_{r+}\left(\frac{K_{\mathrm{eq}}^{A} C_{s}^{A}}{k_{\mathrm{des}}^{B}}+\frac{K_{\mathrm{eq}}^{B} C_{s}^{B}}{k_{\mathrm{des}}^{B}}\right)}{1+K_{\mathrm{eq}}^{A} C_{s}^{A}+K_{\mathrm{eq}}^{B} C_{s}^{B}+k_{r+}\left(\frac{K_{\mathrm{eq}}^{A} C_{s}^{A}}{k_{\mathrm{des}}^{B}}+\frac{K_{\mathrm{eq}}^{B} C_{s}^{B}}{k_{\mathrm{des}}^{A}}+\frac{1}{k_{\mathrm{des}}^{A}}\right)},
\end{aligned}
$$

By comparing these theoretical predictions with SRD results, we can show the validity of the particle-based simulation approach.

\section{Dimensionless analysis}

Mass transport to the catalyst surface and the consecutive processes that occur determine the rate of product formation. This conversion takes into account the presence of other physical parameters, such as the length of the model reactor, the area of the catalyst surface, and bulk concentration of the reactant species. A convenient way to describe these quantities is to define a series of dimensionless numbers that capture the relative strengths of the competing processes involved. A denser and larger system with low catalyst area might give a similar output as a rarer and smaller system but with high catalyst area. Therefore it will be more convenient to represent these systems by the same dimensionless numbers.

Moreover, to facilitate comparison with real experimental systems, in the following sections, we will present all physical quantities in terms of $D_{A}$, the diffusion coefficient of $\mathrm{A}, H$, the height of the model reactor, and $k_{B} T$, the thermal energy. These three dimensions are sufficient to generate all other relevant physical quantities and parameters, as shown in Table III. For example, the dimensionless time becomes $t^{o}=t D_{A} / H^{2}$, the dimensionless velocity $\mathbf{v}^{o}=\mathbf{v} H / D_{A}$, and so on.

\section{Damkohler numbers}

In general, the system complexity will increase with the inclusion of additional intermediary processes as competition between these processes will start. Invariably, only one or two processes determine the rate of the reaction. We will compare

TABLE III. Physical quantities with their dimensions.

\begin{tabular}{lc}
\hline \hline Physical quantities & Dimensions \\
\hline Time & $\frac{H^{2}}{D_{A}}$ \\
Length & $\mathrm{H}$ \\
Energy & $k_{B} T$ \\
Mass & $\frac{k_{B} T H^{2}}{D_{A}^{2}}$ \\
Velocity & $\frac{D_{A}}{H}$ \\
Diffusion & $D_{A}$ \\
Number density & $H^{-3}$ \\
\hline \hline
\end{tabular}


TABLE IV. Flux of product B produced, when each of the mentioned process is rate limiting.

\begin{tabular}{lc}
\hline \hline Rate-limiting process & Flux \\
\hline Adsorption (A) & $k_{\text {ads }}^{A} C_{s}^{A} \theta_{\text {vac }}$ \\
Reaction (A) & $k_{r+} \theta_{A, \text { eq }} N_{\text {cat }}$ \\
Desorption (B) & $k_{\text {des }}^{B} \theta_{B, \text { eq }} N_{\text {cat }}$ \\
\hline \hline
\end{tabular}

these rate-limiting processes with the mass transfer of the reactants to the catalyst surface.

We note that for a first-order Langmuir model (see Sec. III A), it can be imagined that A adsorbs on the catalytic strip, followed by an instantaneous conversion to species $\mathrm{B}$ and desorption of species B. The Langmuir model can then be written as the Langmuir-Hinshelwood model where, $k_{r+} \gg K_{\mathrm{eq}}^{i} C_{s}^{i}$. Since $A \rightarrow B$ occurs almost instantaneously now, the reactive flux is controlled by the adsorptive flux of $\mathrm{A}$ on the strip.

For this approach, Squires et al. [28] has already shown that the diffusive flux is

$$
J_{D} \sim \frac{D_{A}\left(C_{0}^{A}-C_{s}^{A}\right) W H}{\delta},
$$

where $C_{0}^{A}$ is the concentration of solvent in the bulk, $C_{s}^{A}$ is the concentration just near the catalyst surface, and $\delta$ is the boundary layer thickness. $\delta$ is the length it requires for the product particles to diffuse from the source, the catalytic surface, to the exit, the boundary of the reactor along the $z$ direction. Since the catalyst has width $L_{\text {cat }}$ along the $z$ direction, the effective length traversed by product particles before being removed from the system is $\delta=\left(L-L_{\text {cat }}\right) / 2$. The reactive flux (or the adsorptive flux) is given as

$$
J_{R}=k_{\mathrm{ads}}^{A} C_{s}^{A} \theta_{\mathrm{vac}}
$$

where $\theta_{\text {vac }}$ is the fractional vacancy of sites on the catalytic strip at a steady state. At steady-state conditions, the adsorptive and the desorptive fluxes will be equal, leading to

$$
\frac{C_{s}^{A}}{C_{0}^{A}}=\left(1+\frac{k_{\mathrm{ads}}^{A} \theta_{\mathrm{vac}} \delta}{D_{A} W H}\right)^{-1}=\frac{1}{1+\mathrm{Da}} .
$$

The Damkohler number is here defined as

$$
\mathrm{Da}=\frac{k_{\mathrm{ads}}^{A} \theta_{\mathrm{vac}} \delta}{D_{A} W H} .
$$

If $\mathrm{Da} \ll 1, C_{s}^{A} \sim C_{0}^{A}$ and the system is reaction limited. If $\mathrm{Da} \gg 1, C_{s}^{A} \sim 0$ and the system is diffusion limited. Note that $\theta_{\mathrm{vac}}$ is also a function of $C_{s}^{A}$. When $K_{\mathrm{eq}}^{A} C_{s}^{A} \ll 1$, according to Eq. (14), $\theta_{\text {vac }}=1-\theta_{A \text {,eq }} \sim 1$, and the surface will equilibrate quickly enough to enter a quasisteady state [28]. Outside this regime, for a slow equilibration of the surface, the Da analysis mentioned here is still valid. This gives the model a definite edge as we are able to capture phenomena from a linear regime, $K_{\mathrm{eq}}^{A} C_{s}^{A} \ll 1$, to a nonlinear regime, $K_{\mathrm{eq}}^{A} C_{s}^{A} \gg 1$, with the information about the overall reaction order being stored in the expression of $\theta_{\mathrm{vac}}$.

For the Langmuir-Hinshelwood model presented in Eq. (15), when a slow reaction is introduced, the competing processes as stated in Table IV will determine the surface coverages, $\theta_{\mathrm{vac}}, \theta_{A, \text { eq }}, \theta_{B, \text { eq }}$, and eventually the rate of formation of B.

Again, if adsorption of $\mathrm{A}$ is the rate-determining step, the reaction kinetics can be approximated to a Langmuir model and the nonlinearity of the overall reaction can be studied by looking at the magnitude of $K_{\mathrm{eq}}^{A} C_{s}^{A}$.

Desorption of A negatively influences the formation of product B since fewer A* particles are available for conversion. When adsorption of $\mathrm{A}$ is rate determining, and desorption of A also negatively influences the process, the effective rate of reaction becomes $k_{\mathrm{ads}}^{A} C_{s}^{A} \theta_{\mathrm{vac}}-k_{\mathrm{des}}^{A} \theta_{A \text {, eq }}$. Under these conditions equating the reactive flux to diffusive flux gives

$$
\frac{C_{s}^{A}}{C_{0}^{A}}=\frac{1+\frac{k_{\mathrm{des}}^{A} \theta_{A, \mathrm{eq}} N_{\mathrm{cat}} \delta}{C_{0}^{A} D_{A} W H}}{1+\frac{k_{\mathrm{ads}}^{A} \theta_{\mathrm{vac}} \delta}{D_{A} W H}} .
$$

When any other intermediary step determines the rate, we will follow the approach outlined henceforth. If reaction of $A$ is rate limiting, the flux equation is given as

$$
\frac{D_{A}\left(C_{0}^{A}-C s^{A}\right) W H}{\delta}=k_{r+} \theta_{A, \mathrm{eq}} N_{\text {cat }} .
$$

Rewriting this expression gives

$$
\frac{C_{s}^{A}}{C_{0}^{A}}=1-\frac{k_{r+} \theta_{A, \mathrm{eq}} N_{\mathrm{cat}} \delta}{D_{A} C_{0}^{A} W H}=1-\mathrm{Da}_{\mathrm{r}} .
$$

We refer to $\mathrm{Da}_{\mathrm{r}}$ as the modified Damkohler number for the case where the reaction process is the limiting step. The above equation provides a limitation on the expression, $k_{r+} \theta_{A, \text { eq }} N_{\text {cat }} \delta / D_{A} C_{0}^{A} W H<1$, which has to be true always as $J_{D, \text { max }}=D_{A} C_{0}^{A} W H / \delta$ is the maximum possible diffusive flux from the bulk to the surface. If $\mathrm{Da}_{\mathrm{r}} \ll 1$, we can rewrite Eq. (24) as $C_{s} / C_{0}=1 /\left(1+\mathrm{Da}_{\mathrm{r}}\right)$, similar to Eq. (20) but using the modified Damkohler number.

For a slow desorptive process, the relevant Damkohler number can be written as

$$
\mathrm{Da}_{\mathrm{d}}=k_{\mathrm{des}}^{B} \theta_{B, \mathrm{eq}} \delta / D_{A} C_{0}^{A} W H .
$$

Readsorption of B shifts the surface coverage over the catalyst in favor of $\mathrm{B}^{*}$ particles reducing the number of $A^{*}$ particles. This in affect allows for an increase in the production of $B$ particles which get readsorbed over the surface again. Therefore, the effective production of B does not have a strong dependence on $k_{\text {ads }}^{B}$, at least as long as there is no convection. For our analysis which focuses on reaction-diffusion system without convection, we will remain in the regime with $K_{\text {eq }}^{B} \ll 1$, thereby concentrating more on the effect of desorption of $\mathrm{B}$ rather than the adsorption of $\mathrm{B}$.

The dimensionless analysis done here can be used to generalize most of the reaction procedures falling within the Langmuir or the Langmuir-Hinshelwood model. Having described the model and the parameters involved, we now validate the SRD model by comparing results with these theoretical predictions and with previous studies.

\section{MODEL VALIDATION}

Before the introduction of catalyst sites inside the system, we validate the applicability of the model by looking at axial 


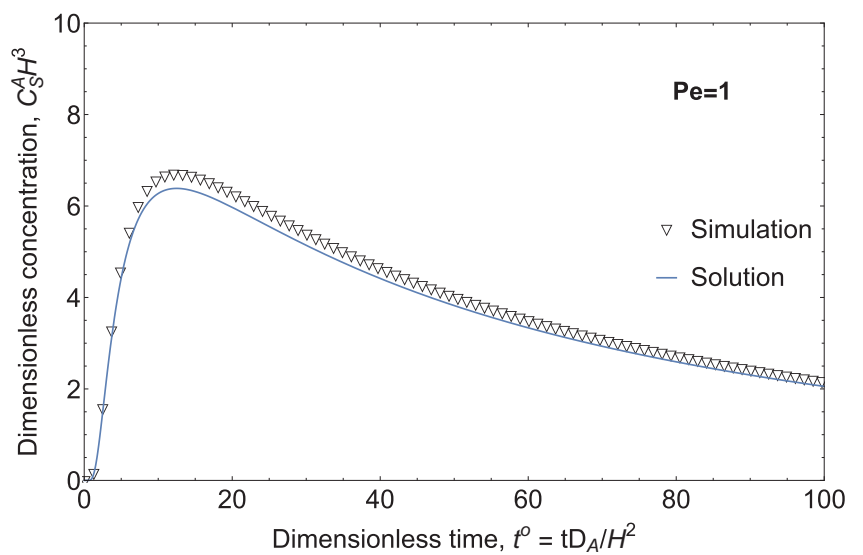

(a)

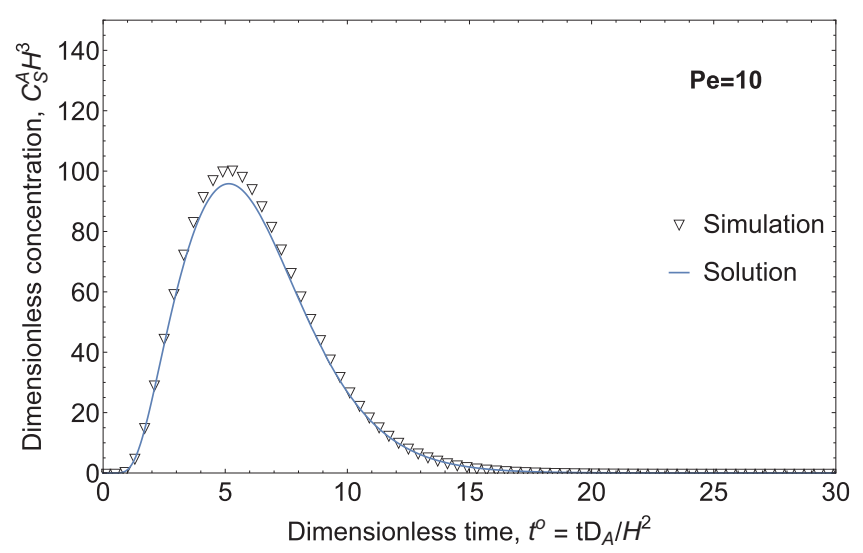

(b)

FIG. 2. Test of axial dispersion of B particles in a solvent of A particles under flow conditions. Here the time dependence of the B-particle concentration in a measurement region in the center of the domain is shown, for $\mathrm{Pe}=1$ (a) and $\mathrm{Pe}=10$ (b). At $t=0, \mathrm{~B}$ particles are introduced in a single time step $\Delta t_{c}$ at the left side of the domain. Inverted triangles represent simulation data, lines are analytical solutions.

dispersion, where convection and diffusion together influence the spatial and temporal evolution of the concentration profile. After this, reactions will be introduced on a catalytic strip.

\section{A. Axial dispersion}

The diffusion coefficient obtained from Eq. (3) can be used for solving the convection-diffusion equation for binary mixtures. Gill and Sankarasubramanian [40] and Kolev et al. [41] have given an exact solution for the parallel plate laminar flow problem for binary systems. For a fully developed laminar profile, with flow occurring in the $z$ direction, the spatial and temporal distribution of concentration of any component (in a binary mixture) will obey the following partial differential equation:

$$
\frac{\partial C_{0}^{i}}{\partial t}+u_{0}^{i}\left[1-\left(\frac{y}{H}\right)^{2}\right] \frac{\partial C_{0}^{i}}{\partial z}-D_{A B} \nabla^{2} C_{0}^{i}=0 .
$$

Here $D_{A B}$ is the Maxwell-Stefan diffusion coefficient for mutual diffusion in a binary mixture. When the diffusivity of both species is the same, $D_{A B}=D_{A}=D_{B}$, or the mutual diffusivity equals diffusivity of either of the components [42]. $C_{0}^{i}$ represents the number density of particles of type $i$, and $u_{0}^{i}$ is the maximum velocity of particles $\mathrm{A}$ or $\mathrm{B}$ in the center of the slit. A solution to Eq. (26) is provided in Ref. [41].

For the SRD model, the solvent particles are the particles A. Initially the system is full of solvent particles A until laminar flow is achieved. This time is set as $t=0$. Following this, B particles, having the same mass as $\mathrm{A}$, are introduced at $t=0$ inside a narrow region from $z=0$ to $z=\alpha L$, where $\alpha=0.05$. This is done by randomly replacing A particles in this narrow region by $B$ particles within a single time step until a number density $C_{0}^{B}$ is achieved for the B particles. For times $t>0$, this tracer region will disperse inside the system due to convection and diffusion. The temporal and spatial concentration of this tracer region has been studied in Ref. [41].

A sensor region is defined in the center of the domain that records the time-dependent number density of particles of type B.
To ensure a particle is not doubly counted in the tracer region (since the system is periodic in the $z$ direction), an artificial coordinate $z_{i, a}$ stores the unfolded $z$ coordinates of B particles such that when a particle crosses a periodic boundary, this coordinate is updated as if it encounters no periodic boundary. This ensures an accurate calculation of particles present in the tracer region at any time as $z_{i, a}$ acts as a numerical check as to whether particles are entering the tracer region for the first time.

We now look at the temporal concentration profile of $B$ particles measured in the tracer region for two cases with different Péclet number. The Péclet number is defined as $\mathrm{Pe}=$ $L u / 2 D_{B}$. Here $L / 2$ is the length along the $z$ axis from $z=0$ until the position of sensor, $u$ is the average $z$ velocity of particles B, i.e., $2 u_{0}^{B} / 3$, and $D_{B}$ is the diffusion coefficient of particles B.

In Figs. 2(a) and 2(b), the temporal concentration profile of particles has been plotted for the case aforementioned. There is a time lag observed before any concentration is recorded. This dead zone is the time taken for the fastest particles to reach the tracer region. The peak observed represents the most probable time taken by particles to reach the sensor from the start of the system, $t_{m p}=u / 3 L$, which is the time required by any particle with velocity equal to the average velocity $2 u_{0}^{B} / 3$ to cover the distance $L / 2$. The simulation results (inverted triangles) are compared with the analytical formula mentioned in Ref. [41] (lines). We find a good agreement between the simulation model predictions and the analytical formula, indicating that convection and diffusion are correctly modeled. Next we validate the model for heterogeneous reactions and diffusion.

\section{B. Adsorption-desorption reaction}

With the introduction of a catalytic strip, as described in Sec. II B, the time evolution of the surface coverage study is a matter of interest. For a system consisting entirely of A particles with number density $C_{0}^{A}$, when adsorption starts, the adsorption rate is $k_{\text {ads }}^{A} C_{0}^{A}$. As adsorption and subsequent processes progress towards a steady state, a fraction of particles remain on the catalyst strip at all times. Even for a first-order 
Langmuir model, with no reaction, this results in a change in local number density $C_{s}^{A}$, which is different from $C_{0}^{A}$.

For a first-order Langmuir model, the surface coverage of A, $\theta_{A \text {,eq }}$ was calculated with the simulations and compared with Eq. (14). For low to moderate probabilities of desorption, the results are in agreement with the expression. However, for higher value of $p_{\mathrm{des}}^{A}$, i.e., $p_{\mathrm{des}}^{A}>0.5$, discrepancies were recorded in the calculation of $\theta_{A, \text { eq }}$. This was found to be caused by the discretized implementation of adsorption and desorption in the model.

For a given time step, all particles that could be adsorbed were allowed to adsorb on the strip, thus increasing $\theta_{A}$. This was immediately followed by desorptions from the strip. To demonstrate how this approach changes $\theta_{A \text {, eq }}$, let us consider an example with a strip comprised of $N_{\text {cat }}=1000$ catalytic sites. Let 100 particles be permitted to be adsorbed on this strip at a time step. Therefore, at this time step, $\theta_{A}$ increases by a value of 0.1 before starting desorptions. When desorption is tried, the number of particles on the catalytic strip is higher than would have occurred had desorption been taking place simultaneously with the adsorption [43]. This effect grows for higher $p_{\text {des }}$, and the $\theta_{A \text {,eq }}$ obtained in this case is lower than what is expected.

The above issue arises because of the treatment of adsorption and desorption processes sequentially instead of simultaneously. The difference can be made arbitrarily small (for fixed adsorption and desorption rates) by subdividing the simulation time step $\Delta t_{c}$ in a number of smaller steps with alternating adsorption and desorption steps. Here we propose another approach, by which the same time step $\Delta t_{c}$ can be retained. To this end, we rewrite the differential equation for $\theta_{A}[$ Eq. (14)] as

$$
\frac{d \theta_{A}}{d t}=k_{\mathrm{ads}}^{A} C_{s}^{A}\left(1-\theta_{A}\right)\left[1-\frac{k_{\mathrm{des}}^{A}}{k_{\mathrm{ads}}^{A} C_{s}^{A}\left(1-\theta_{A}\right)} \theta_{A}\right] .
$$

The equation can be interpreted as follows. For every particle adsorbed, a subsequent $k_{\mathrm{des}}^{A} \theta_{A} / k_{\mathrm{ads}}^{A} C_{s}^{A}\left(1-\theta_{A}\right)$ number of particles will be desorbed. As $k_{\mathrm{des}}^{A} \theta_{A} / k_{\mathrm{ads}}^{A} C_{s}^{A}\left(1-\theta_{A}\right)$ tends to 1 , the system approaches equilibrium. Thus, equilibrium is said to be achieved when for every adsorption, there is
TABLE V. Observed values of $\theta_{A \text {, eq }}$ for increasing $p_{\text {des }}^{A}$. Parameter set used: $k_{B} T=1.0, \Delta t_{c}=1.0, m_{A}=1.0, \gamma=8, A_{\text {cat }}=2.0, p_{\text {on }}^{A}=$ $1.0, N_{\text {cat }}=100$.

\begin{tabular}{ccccc}
\hline \hline$p_{\text {des }}^{A}$ & $K_{\text {eq }}^{A} C_{s}^{A}$ & $\theta_{A, \text { eq }}$ Eq. (14) & $\theta_{A, \text { eq }}$ Eq. (12) & $\theta_{A, \text { eq }}$ Eq. (27) \\
\hline 0.1 & 1.348 & 0.574 & 0.571 & 0.572 \\
0.3 & 0.398 & 0.285 & 0.278 & 0.283 \\
0.5 & 0.205 & 0.170 & 0.16 & 0.167 \\
0.7 & 0.124 & 0.105 & 0.092 & 0.101 \\
\hline \hline
\end{tabular}

a corresponding desorption. By rewriting the expression for $d \theta_{A} / d t$ as given by Eq. (27), the analytically expected results are more accurately reproduced, as shown in Table V.

It has to be mentioned that there is an inherent error percentage attached to this solution, which is of the order of magnitude of $1 / N_{\text {cat }} \times 100$. Increasing $N_{\text {cat }}$ will reduce this error but increase the computational time. Therefore it is imperative that we select values of $N_{\text {cat }}$ with error tolerance that do not hinder the model computationally.

The rate equation for the Langmuir-Hinshelwood reaction model [Eq. (15)] can similarly be rewritten to get a sequential treatment of processes which resembles the simultaneous processes better. The new rate equation is

$$
\begin{aligned}
\frac{d \theta_{A}}{d t} & =k_{\mathrm{ads}}^{A} C_{s}^{A} \theta\left(1-\frac{k_{\mathrm{des}}^{A}}{k_{\mathrm{ads}}^{A} C_{s}^{A} \theta} \theta_{A}-\frac{k_{r+}}{k_{\mathrm{ads}}^{A} C_{s}^{A} \theta} \theta_{A}\right), \\
\frac{d \theta_{B}}{d t} & =k_{r+} \theta_{A}\left(1-\frac{k_{\mathrm{des}}^{B}}{k_{r+} \theta_{A}} \theta_{B}+\frac{k_{\mathrm{ads}}^{B} C_{s}^{B}}{k_{r+} \theta_{A}} \theta\right) .
\end{aligned}
$$

For every adsorption of A, there are $k_{\mathrm{des}}^{A} \theta_{A} / k_{\mathrm{ads}}^{A} C_{s}^{A} \theta$ desorptions of $\mathrm{A}^{*}$ and $k_{r+} \theta_{A} / k_{\text {ads }}^{A} C_{s}^{A} \theta$ conversions from $\mathrm{A}^{*}$ to $\mathrm{B}^{*}$. Subsequently, for every conversion, there are $k_{\mathrm{des}}^{B} \theta_{B} / k_{r+} \theta_{A}$ desorptions of $\mathrm{B}^{*}$ and $k_{\mathrm{ads}}^{B} C_{s}^{B} \theta / k_{r+} \theta_{A}$ readsorptions of $\mathrm{B}$.

In summary, by rewriting the continuum equations, we can deduce how to treat adsorption, reaction and desorption processes in a sequential fashion. We are now ready to study reaction-diffusion systems for which analytical solutions are unavailable.

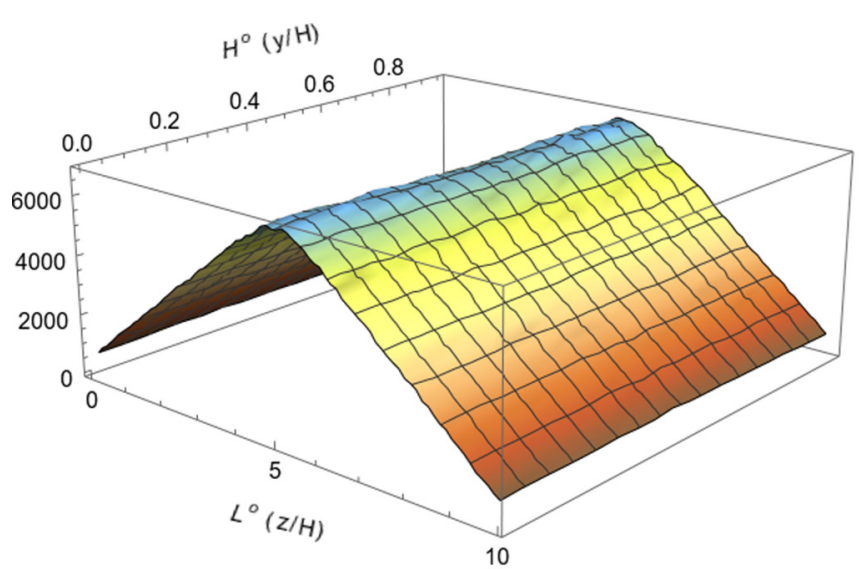

(a)

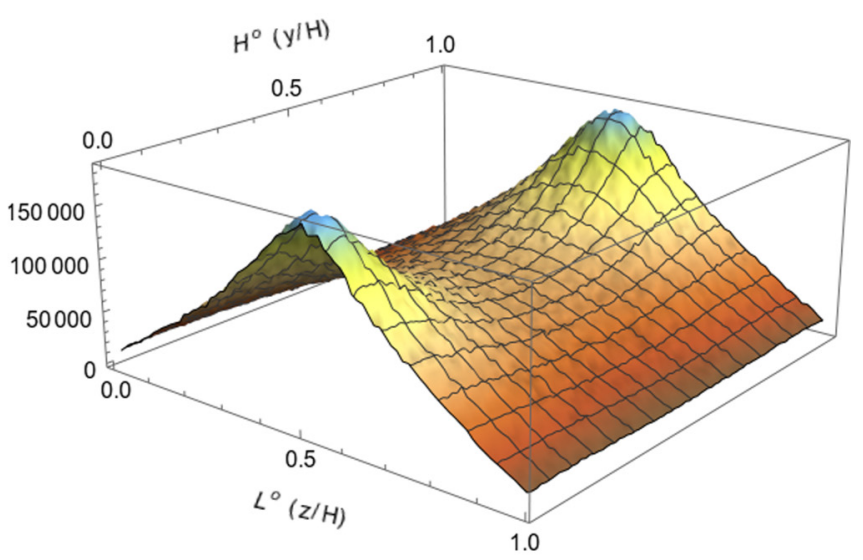

(b)

FIG. 3. Product concentration profiles along the $z$ and $y$ directions of the model reactor for (a) small and (b) large wall-to-wall distance $H$ at steady state. The bottom and top walls are located along $y^{o}=0(H=4)$ and $y^{o}=1(H=40)$, respectively. $\gamma=100, \Delta t_{c}=1.0 p_{\text {ads }}^{A}=1.0$, $p_{\mathrm{des}}^{A}=0.02, p_{r+}=0.9, p_{\mathrm{des}}^{B}=0.7, p_{\mathrm{ads}}^{B}=0.01$. 


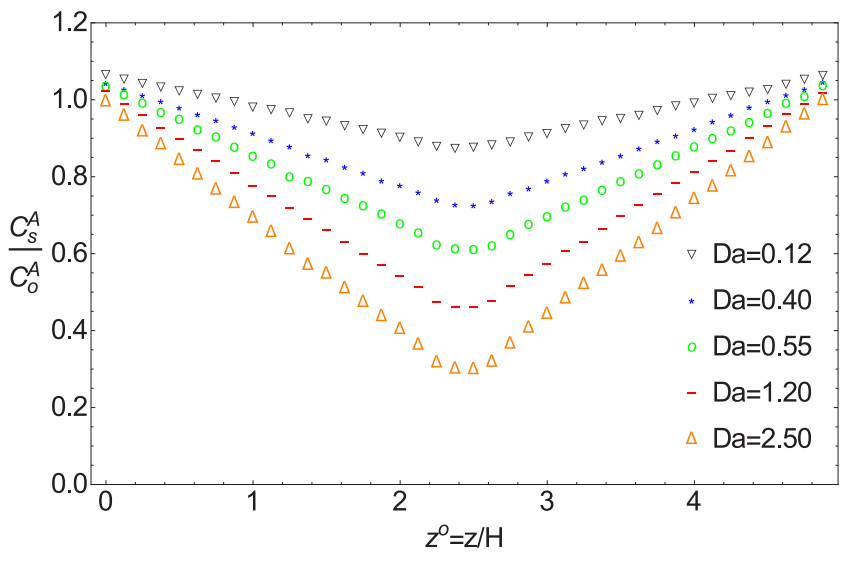

(a)

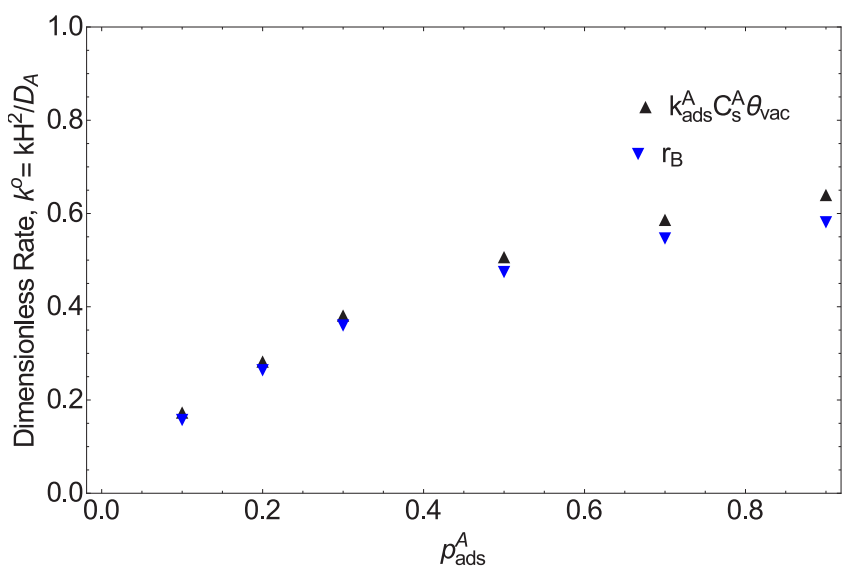

(b)

FIG. 4. Adsorption of $\mathrm{A}$ is the rate-determining step, so the rate of formation of $\mathrm{B}$ is controlled by A adsorbing on the surface. (a) The depletion in local concentration of $\mathrm{A}$, at steady state, across the reactor length where the catalytic strip is present at the center of the system. $\mathrm{Da}$ is varied by changing the probability of adsorption of A. (b) At steady state, the production rate of $\mathrm{B}$ and adsorption rate of $\mathrm{A}$ are compared. The parameter set used is $\gamma=10, \Delta t_{C}=1.0, p_{\text {des }}^{A}=0.1$, $p_{r+}=0.7, p_{\text {des }}^{B}=0.7, H=4, W^{o}=W / H=0.5, L^{o}=L / H=5$, $L_{\text {cat }}^{o}=L_{\text {cat }} / H=0.5, N_{\text {cat }}=1000$.

\section{RESULTS}

Reactions occur at the center of the system on the catalytic strip. The product particles B diffuse out into the bulk of the system after desorption. At $z=0$ and $z=L$, particles $\mathrm{B}$ are removed from the system. A concentration profile for both reactants and products is formed with product concentration going to 0 at the ends of the system.

Here we will consider a pseudo-one-dimensional (1D) case where we examine the concentration gradient along a single direction while there is effectively no gradient present in the other two directions. To achieve this, we place an identical catalyst along the wall at $y=H$ sharing the same $x$ and $z$ coordinates.

Figures 3(a) and 3(b) show that the distance $H$ between the two strips is relevant for replicating a pseudo-1D model. Once product B is desorbed back into the system, it starts diffusing from near the strip in all three directions. Because

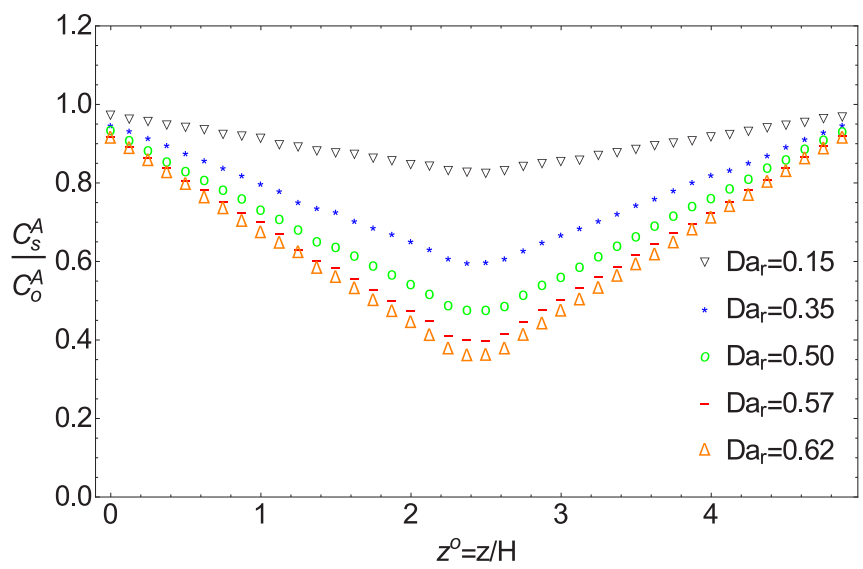

(a)

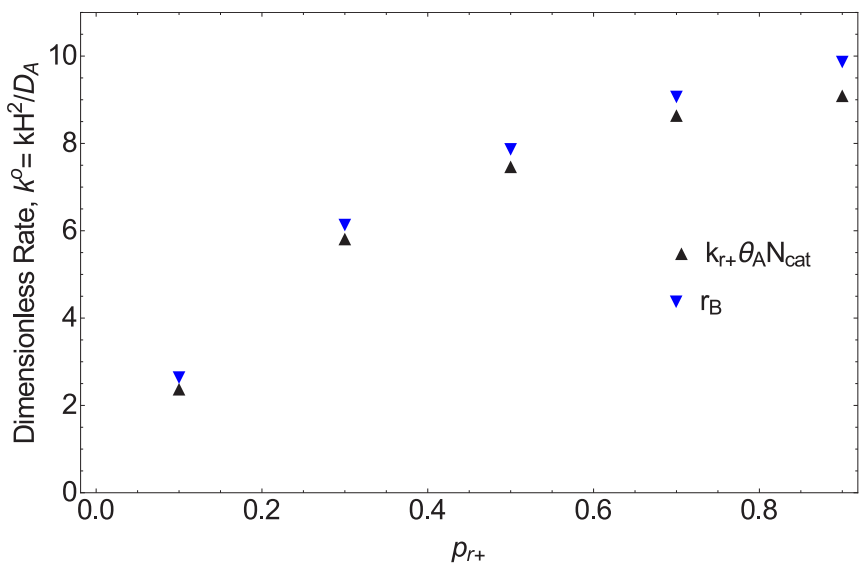

(b)

FIG. 5. Reaction of $\mathrm{A}$ is the rate determining step. (a) The depletion in local concentration of $\mathrm{A}$, at steady state, across the reactor length. $\mathrm{Da}_{\mathrm{r}}$ is varied by changing the probability of reaction of $\mathrm{A}$. (b) At steady state, the production rate of $\mathrm{B}$ and reaction rate of $\mathrm{A}$ are compared. The parameter set used is the same as in Fig. 4 except $p_{\mathrm{on}}^{A}=1.0, N_{\mathrm{cat}}=100, \gamma=100 . p_{r+}$ is varied from 0.1 to 0.9 .

the catalytic strip covers the entire width $W$ in the $x$ direction there will be no concentration gradient along this direction. If the wall-to-wall distance $H$ is sufficiently small, gradients in the $y$ direction will also be small. The following results are for such a pseudo-1D system where $z$ is the only relevant coordinate.

In Fig. 4(a) the number density profile across the length of the reactor in the $z$ direction is shown. For increasing Da, the dip in $C_{s}^{A}$ deepens representing an increasing concentration of reactant getting extinguished near the surface as the probability of adsorption for particles A increases.

In Fig. 4(b) the rate of adsorption of A and generation of B is plotted for different probabilities of adsorption of A. As $p_{\mathrm{on}}^{A}$ increases, the surface coverage $\theta_{A}$ will increase. This will, in turn, cause an increase in the desorption of particles from the surface, and therefore the net production of B will effectively be the difference between these two rates, as mentioned in Sec. III C 1. The production of B is also seen to move from a linear regime, for low $p_{\text {ads }}^{A}$ to a nonlinear regime for higher $p_{\text {des }}^{A}$ as was also discussed in Sec. III C 1. 


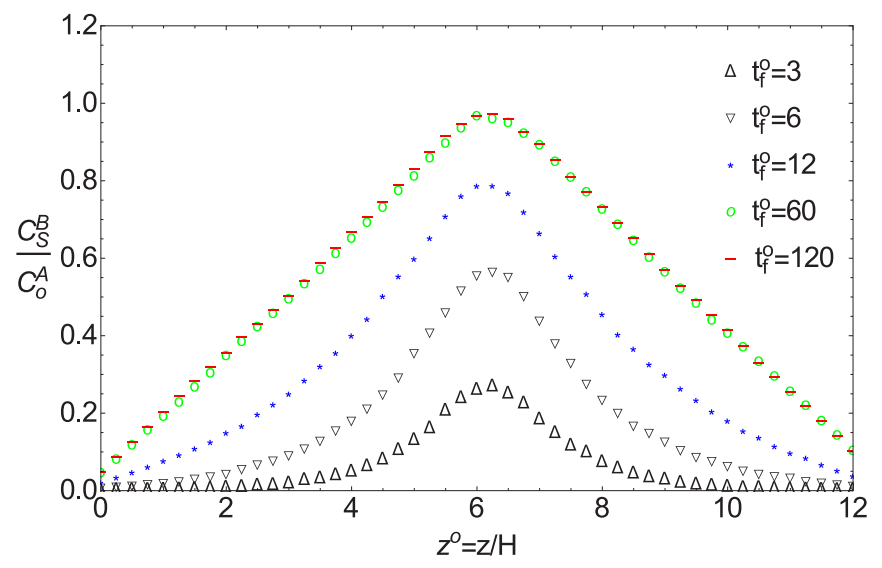

FIG. 6. Product concentration profile across the length of the reactor for different simulation times, $t_{f}$. The dimensionless time, $t_{f}^{o}=t_{f} D_{A} / H^{2}$.

A similar analysis has been done for the case when the reaction rate becomes rate determining. Figure 5(a) shows the variation of local number density of $A$ across the length of the reactor for different $\mathrm{Da}_{\mathrm{r}}$ by changing the probability of reaction. The modified Damkohler number $\mathrm{Da}_{\mathrm{r}}$ can have a maximum value of 1 . In Fig. 5(b), the reaction rate and rate of production of $\mathrm{B}$ have been plotted against the probability of reaction, $p_{r++}$. For low probabilities of reaction, the surface coverage of $\mathrm{B} *$ is still low, causing a lower production rate of $\mathrm{B}$, and thus readsorption of $\mathrm{B}$ is not able to influence the reaction kinetics much. However, with an increase in reaction probability, $\mathrm{B}^{*}$ coverage increases, increasing the local concentration of B over the catalyst. Now $K_{\mathrm{eq}}^{B} C_{s}^{B}$ becomes dominant enough to play a part in the overall rate of production of $B$, which now consists of the reactive term and the back-adsorption term. Therefore, we see a net production rate of B slightly higher than the net reaction rate occurring over the catalyst.

The two above cases expand the model applicability to cases where multiple processes can compete to determine the overall rate of production.

The evolution of the concentration profile of B across the length of the reactor is a process governed by diffusion entirely. The number density depends on the effective rate of the reaction, but the time required to achieve a linear profile is a diffusive process. The evolution happens according to Fick's second law, generating a error function solution of the number density profile. For large reactor length, the diffusive time is of the order of $t_{D}=L^{2} / D_{B}$. In such cases, we need to make sure that the simulations are run for a sufficiently long time to achieve the steady state. Figure 6 shows the evolution of the concentration profile of product particles inside the bulk. The half maxima of the temporally varying concentration profile was found to be proportional to $\sqrt{D_{B} t}$, which is to be expected.

\section{CONCLUSION}

Stochastic rotation dynamics has been used to bridge the gap between the macroworld and the microworld by simulating reactions at a catalytic surface with the products diffusing back into the bulk of the system. The microworld knowledge about the reaction has been used in the form of reaction models, and the macroworld knowledge is obtained by looking at the concentration profiles of the reactant and products obtained due to the reaction.

We have outlined how a multicomponent SRD model can be extended to describe heterogeneous reactions with rate-limiting steps, such as adsorption, desorption, and slow reactions, to observe real physical behavior for such mixtures near the catalytic surface. This was implemented through a special treatment of boundary cells. A reactor system was modeled with the presence of a catalytic strip inside the system. Adsorption on this strip would either result in desorption after some time (Langmuir model) or lead to an irreversible reaction leading to product generation (LangmuirHinshelwood model). A parametric study was done to calculate the different rates of adsorption, desorption, and reaction in terms of the simulation parameters derived for the SRD model in previous works.

A general approach to model simultaneous processes using particle-based methods was outlined for a simple adsorption desorption model, which was further extended to include a reaction. At a microlevel, processes like adsorption, desorption, and reaction do happen discretely. However, when considering these processes within a mean-field approximation, we need to rely on methods that will treat these processes simultaneously.

Incorporating a reaction model involves numerous subprocesses that compete to determine the overall concentration profile of products in the bulk. The number of these subprocesses will just increase with the increase in number of components involved in the reaction. To facilitate this calculation, we resorted to the use of a dimensionless analysis for all the physical quantities and rate parameters. Damkohler numbers were used to calculate the amount of reactant that will be depleted at the catalyst surface. Three different Damkohler numbers were defined in this work, depending on the ratelimiting process, that stand as a measure of the flux of product generated to the flux of products diffusing out of the system. Given the reaction and simulation parameters combined with these Damkohler numbers, the surface coverage of different component species over the catalyst can be measured. This can in turn be used to estimate the effective reaction order.

We ended the paper by analyzing the spatial and temporal distribution of the concentration of product species across the model system, and we interpreted the results using the Damkohler numbers derived in the previous sections. We also commented on the influence of processes like desorption of reactant species from the catalytic surface and readsorption of product species back on the surface on the overall rate of production of products. As soon as products are desorbed back into the system, it is the diffusive process that takes them to the system boundaries where they are eventually extracted. We analyzed the time needed for this process to reach steady-state values across the bulk of the reactor system.

In our future work, we will include more complex reactions and allow for different mobilities of the participating components in the presence of an external body force. This will allow us to model certain real-life reactive systems, capturing the coupled hydrodynamics and reaction kinetics efficiently. 


\section{ACKNOWLEDGMENTS}

The work is part of the Netherlands Center for Multiscale Catalytic Energy Conversion (MCEC), funded by the Netherlands Organisation for Scientific Research, NWO. A.S. thanks B. Fitzgerald for a careful reading of the manuscript.

\section{APPENDIX A: BOUNDARY-FILLING RULE}

For the simulation box with walls along $y=0$ and $y=H$, the random grid shift procedure leads to an artificially low number of particles in the grid cells overlapping with the boundary, which we will refer to as boundary cells. This artifact leads to a lower viscosity region along these boundaries, leading to an effective slip layer. For a multicomponent fluid, $m_{\text {bulk }}=\sum_{i=1}^{k} \chi_{i} m_{i} N / V$ represents the average mass inside a bulk grid cell for a $k$-component mixture. If the mass $m^{\prime}$ recorded inside the boundary cell is less than $m_{b u l k}$, extra mass has to be imparted to this cell to account for the collisional part of the model. This can be done by adding the fluctuating momentum of a single particle of mass $\left(m_{b u l k}-m^{\prime}\right)$ to the total momentum of the boundary grid cell. This is simply a Maxwell-Boltzmann distributed momentum of variance $\left(m_{\text {bulk }}-m^{\prime}\right) k_{B} T$. If $N(0,1)$ is a Gaussian random number with mean 0 and 1 variance, $N\left(0, k_{B} T /\left(m_{\text {bulk }}-m^{\prime}\right)\right.$ will be the random number that gives velocity of this artificial particle. The modified net momentum of the grid cell will be

$$
\mathbf{m v}_{f}=\mathbf{m v}_{i}+\left(m_{b u l k}-m^{\prime}\right) N\left(0, \frac{k_{B} T}{m_{b u l k}-m^{\prime}}\right) .
$$

The center of mass velocity $\mathbf{m} \mathbf{v}_{f} / m_{\text {bulk }}$ calculation is proceeded by the collisional step to get new velocities.

\section{APPENDIX B: ADSORPTION-DESORPTION TREATMENT}

When no reaction occurs inside the system, the collisions at a boundary take place with a no-stick boundary condition. Adsorption of particles leading to reaction and further desorption of product particles involves a certain amount of time for which these particles remain at the surface. It is therefore necessary to simulate the desorption process effectively to maintain a net zero velocity at the boundaries without changing the particle interaction dynamics.

This is accomplished by matching the velocity distribution of desorbing particles with that of the incoming particles. The tangential component of velocity imparted to the desorbed particles is the same as that of the incoming particles whereas the normal component is just the reverse. For our particular system with walls along the $y$ direction, the velocities of desorbing particles along the $x$ and $z$ directions follow a Maxwell-Boltzmann distribution with mean 0 and a standard deviation $\sqrt{k_{B} T / m}$. However, the $y$ component of the velocity of the incoming particles collected during a time interval $\Delta t_{c}$ has a bias. Atkins [35] showed that this distribution in the

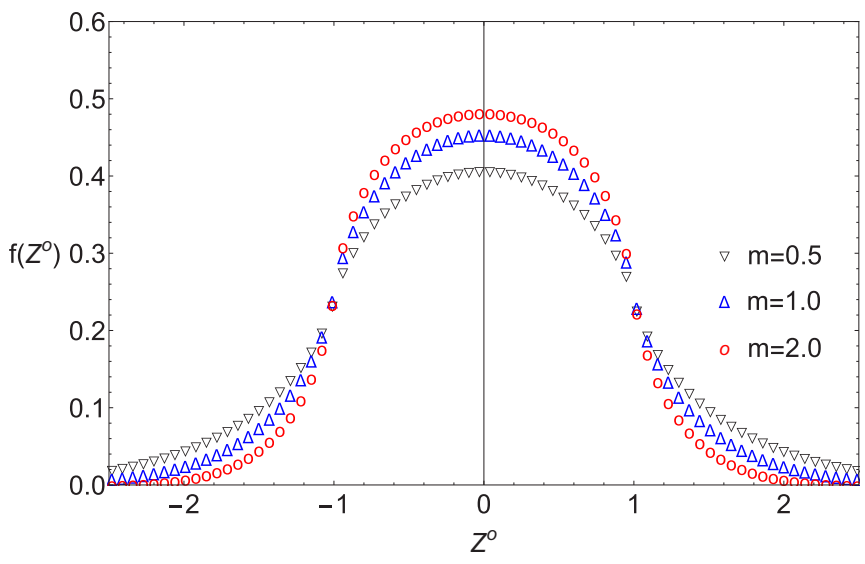

FIG. 7. Probability distribution of the $z$ position of particles desorbed within a time step $\Delta t_{c}$ for different particle masses. The catalyst is placed at the center of a system with a $L_{\text {cat }}=2.0$, $\Delta t_{c}=1.0$.

normal direction is given by

$$
P\left(v_{y}\right)=\frac{m}{k_{B} T} v_{y} e^{-m v_{y}^{2} / 2 k_{B} T} .
$$

A particle desorbs back into the system from its position at the catalytic site into the bulk fluid. Particle desorption can occur any time between the discrete time step $\left(t, t+\Delta t_{c}\right)$, therefore we choose a random time from the interval $\left[0, \Delta t_{c}\right]$ and displace the particle with its new velocity over this time.

In the case under discussion, the two catalysts are rectangular strips present homogenously along the $x$ direction and running from $L / 2-L_{\text {cat }} / 2$ to $L / 2+L_{\text {cat }} / 2$ along the $z$ direction, with the separation $H$ between them in the $y$ direction effectively small. We therefore expect the product formed to be uniformly distributed along the $x$ and $y$ directions and with a gradient in the $z$ direction.

Figures 7 and 8 show the probability distribution of the $z$ position and $y$ position of particles after desorption. In Fig. 7 the catalytic strip is present along $Z^{o} \in(-1,1)$. In Fig. 8 the catalyst is present at $Y^{o}=0$.

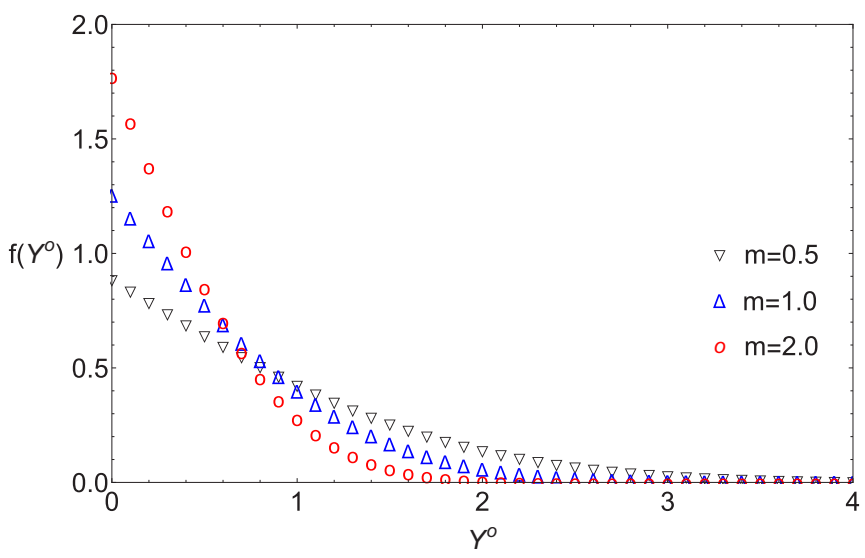

FIG. 8. Probability distribution of the $y$ position of particles desorbed within a time step $\Delta t_{c}$ for different particle masses. $\Delta t_{C}=1.0$. 
[1] R. G. Parr and W. Yang, Density Functional Theory of Atoms and Molecules, 9th ed. (John Wiley \& Sons, New York, 1993), Vol. 47, p. 333.

[2] J. Callaway and N. H. March, Solid State Physics, 38th ed., edited by H. Ehrenreich and D. Turnbull (Academic Press, Orlando, 1984), p. 135.

[3] J. A. Dumesic, D. F. Rudd, L. M. Aparicio, J. M. Rekoske, and A. A. Trevino, The Microkinetics of Heterogeneous Catalysis (American Chemical Society, Washington, DC, 1993), p. 315.

[4] A. Munjiza, The Combined Finite-Discrete Element Method (Wiley, New York, 2004), p. 333.

[5] S. A. Orszag, J. Fluid Mech. 41, 363 (1970).

[6] A. Malevanets and R. Kapral, J. Chem. Phys. 110, 8605 (1999).

[7] E. Allahyarov and G. Gompper, Phys. Rev. E 66, 036702 (2002).

[8] T. Ihle and D. M. Kroll, Phys. Rev. E 67, 066705 (2003).

[9] E. Tüzel, M. Strauss, T. Ihle, and D. M. Kroll, Phys. Rev. E 68, 036701 (2003).

[10] C. M. Pooley and J. M. Yeomans, J. Phys. Chem. B 109, 6505 (2005).

[11] E. Tüzel, T. Ihle, and D. M. Kroll, Phys. Rev. E 74, 056702 (2006).

[12] N. Kikuchi, C. M. Pooley, J. F. Ryder, and J. M. Yeomans, J. Chem. Phys. 119, 6388 (2003).

[13] G. Gompper, T. Ihle, D. M. Kroll, and R. G. Winkler, in Advanced Computer Simulation Approaches for Soft Matter Sciences III (Springer, Berlin, 2009), pp. 1-87.

[14] H. Noguchi and G. Gompper, Phys. Rev. E 78, 016706 (2008).

[15] A. Malevanets and Yeomans, Europhys. Lett. 52, 231 (2000).

[16] M. Ripoll, K. Mussawisade, R. G. Winkler, and G. Gompper, Europhys. Lett. 68, 106 (2007).

[17] E. Tüzel, G. Pan, T. Ihle, and D. M. Kroll, Europhys. Lett. 80, 40010 (2007).

[18] J. T. Padding and A. A. Louis, Phys. Rev. E 74, 031402 (2006).

[19] H. Noguchi and G. Gompper, Phys. Rev. E 72, 011901 (2005).

[20] J.-X. Chen and R. Kapral, J. Chem. Phys. 134, 044503 (2011).

[21] K.-W. Lee and M. G. Mazza, J. Chem. Phys. 142, 164110 (2015).

[22] K. Rohlf, S. Fraser, and R. Kapral, Comput. Phys. Commun. 179, 132 (2008).
[23] K. Tucci and R. Kapral, J. Chem. Phys. 120, 8262 (2004).

[24] C. Echeverria and R. Kapral, Physica D 239, 791 (2010).

[25] S. Thakur and R. Kapral, J. Chem. Phys. 135, 024509 (2011).

[26] M.-J. Huang, J. Schofield, and R. Kapral, Soft Matter 12, 5581 (2016).

[27] S. P. Dawson, S. Chen, and G. D. Doolen, J. Chem. Phys. 98, 1514 (1993).

[28] T. M. Squires, R. J. Messinger, and S. R. Manalis, Nat. Biotechnol. 26, 417 (2008).

[29] J. J. Carberry, Chemical and Catalytic Reaction Engineering (McGraw-Hill, New York, 1976), p. 642.

[30] A. Lamura, G. Gompper, T. Ihle, and D. M. Kroll, Europhys. Lett. 56, 319 (2001).

[31] J. K. Whitmer and E. Luijten, J. Phys.: Condens. Matter 22, 104106 (2010).

[32] R. Kapral, Multiparticle Collision Dynamics: Simulation of Complex Systems on Mesoscales, Technical Report (2008).

[33] K. J. Laidler and M. C. King, J. Phys. Chem. 87, 2657 (1983).

[34] A. van de Runstraat, J. van Grondelle, and R. A. van Santen, Ind. Eng. Chem. Res. 36, 3116 (1997).

[35] P. Atkins and J. de Paula, Physical Chemistry for the Life Sciences, 2nd ed. (Oxford University Press, Oxford, 2006).

[36] G. A. Somorjai, Introduction to Surface Chemistry and Catalysis (Wiley, New York, 1994), p. 667.

[37] G. B. Marin and G. S. Yablonsky, Kinetics of Chemical Reactions (Wiley-VCH, Weinheim, 2011), p. 428.

[38] R. I. Masel, Principles of Adsorption and Reaction on Solid Surfaces (Wiley, New York, 1996), p. 804.

[39] R. A. van Santen and J. W. Niemantsverdriet, Chemical Kinetics and Catalysis (Plenum, New York, 1995).

[40] W. N. Gill and R. Sankarasubramanian, Proc. R. Soc. London A 316, 341 (1970).

[41] S. D. Kolev and W. E. van der Linden, Analyt. Chim. Acta 247, 51 (1991).

[42] R. Krishna and J. Wesselingh, Chem. Eng. Sci. 52, 861 (1997).

[43] For a macroworld, things appear to happen simultaneously, but if you look at it on a nanolevel, the individual processes will be discrete. 